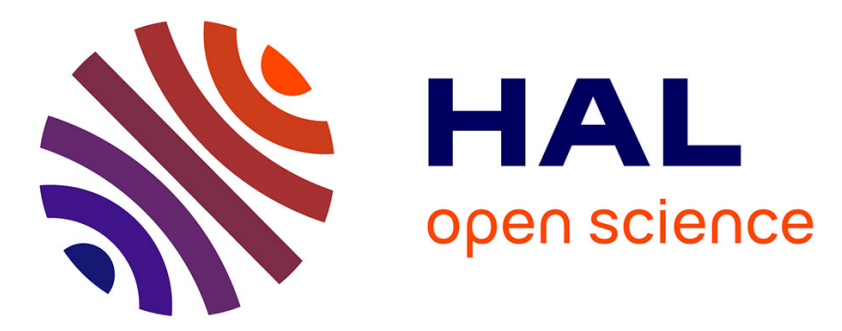

\title{
Relation entre la turbidité et les matières en suspension en réseau d'assainissement unitaire
}

\author{
Ali Hannouche, Ghassan Chebbo, Gwenaël Ruban, Bruno Tassin, Claude
}

Joannis

\section{- To cite this version:}

Ali Hannouche, Ghassan Chebbo, Gwenaël Ruban, Bruno Tassin, Claude Joannis. Relation entre la turbidité et les matières en suspension en réseau d'assainissement unitaire. Techniques Sciences Méthodes , 2011, 2010 (10), pp.42-51. hal-00722663

\section{HAL Id: hal-00722663 \\ https://hal-enpc.archives-ouvertes.fr/hal-00722663}

Submitted on 3 Aug 2012

HAL is a multi-disciplinary open access archive for the deposit and dissemination of scientific research documents, whether they are published or not. The documents may come from teaching and research institutions in France or abroad, or from public or private research centers.
L'archive ouverte pluridisciplinaire HAL, est destinée au dépôt et à la diffusion de documents scientifiques de niveau recherche, publiés ou non, émanant des établissements d'enseignement et de recherche français ou étrangers, des laboratoires publics ou privés. 


\begin{tabular}{|c|c|}
\hline $\begin{array}{l}\text { A. Hannouche } e^{*, * *} \\
\text { G.Chebbo }{ }^{*, * * * *} \\
\text { G. Ruban } \\
\text { B. Tassin }{ }^{* * *} \\
\text { C. Joannis }\end{array}$ & $\begin{array}{l}\text { Relation entre la turbidité et la } \\
\text { concentration en Matières En Suspension } \\
\text { en réseau d'assainissement unitaire } \\
\text { Relationship between turbidity and } \\
\text { suspended solids concentration within } \\
\text { a combined sewer system }\end{array}$ \\
\hline $\begin{array}{l}\text { * Université Paris-Est, LEESU, UMR-MA- } \\
\text { 102, AgroParisTech, } 6 \text { et } 8 \text { avenue Blaise } \\
\text { Pascal - Cité Descartes, } 77455 \text { Champs-sur- } \\
\text { Marne Cedex 2, France } \\
\text { ** SEPIA Conseils, } 53 \text { rue de Turbigo, } 75003 \\
\text { - Paris - France } \\
\text { *** LCPC - Division eau \& environnement - } \\
\text { route de Bouaye - BP } 4129 \text { - } 44341 \\
\text { Bouguenais cedex } \\
\text { **Faculté de génie, Université Libanaise, } \\
\text { route de l'aéroport, Beyrouth, Liban }\end{array}$ & $\begin{array}{l}\text { Table des matières : } \\
\text { INTRODUCTION } \\
\text { METHODOLOGIE } \\
\text { RESULTATS ET DISCUSSIONS } \\
\text { CONCLUSIONS } \\
\text { REMERCIEMENTS } \\
\text { BIBLIOGRAPHIE }\end{array}$ \\
\hline
\end{tabular}

MOTS-CLÉS : étalonnage, concentration en MES, temps sec, temps de pluie, turbidité, variabilité. KEYWORDS: calibration, TSS concentration, dry weather, wet weather, turbidity, variability.

\section{RESUME}

Cet article confirme l'existence d'une relation linéaire forte entre la turbidité et la concentration en Matières En Suspension (MES). Toutefois, la pente de cette relation varie entre le temps sec et le temps de pluie, ainsi que entre les sites. L'effet de cette variabilité sur l'estimation de la concentration en MES par temps de pluie est évalué en fonction du nombre de données d'étalonnage utilisées pour établir la relation entre la turbidité et les MES. Les résultats obtenus montrent à la fois une variabilité limitée entre les sites étudiés et par temps sec, avec une variabilité interévénemtielle significative. De plus, la turbidité permet une évaluation de la concentration en MES avec une précision acceptable pour un effort d'échantillonnage d'événements pluvieux raisonnable.

\section{ABSTRACT}

This article confirms the existence of a strong linear relationship between turbidity and suspended solids (TSS) concentration. However, the slope of this relation varies between dry and wet weather conditions, as well as between sites. The effect of this variability on estimating the instantaneous wet weather TSS concentration is assessed on the basis of the size of the calibration dataset used to establish the turbidity TSS relationship. Results obtained indicate limited variability both between sites and during dry weather, along with a significant inter-event variability. Moreover, turbidity allows an evaluation of TSS concentrations with an acceptable level of accuracy for a reasonable rainfall event sampling campaign effort.

\section{INTRODUCTION}

Depuis 1970, de nombreuses études ont souligné l'importance de la pollution des rejets urbains de temps de pluie et l'impact négatif de cette pollution sur les milieux récepteurs (Ellis et Hvitved Jacobsen, 1996, Gromaire et al., 2001, Saget et al., 1995, Smullen et al., 1999). Les matières en suspension (MES) constituent le principal vecteur des polluants transportés par temps de pluie dans le réseau d'assainissement unitaire (Ashley et al., 2005, Chebbo et al., 1995). Les dispositions réglementaires émanant de la loi sur l'eau du 30 décembre 2006 imposent le traitement des effluents pour les événements non exceptionnels et l'autosurveillance des ouvrages de collecte et de traitement. De plus, dans le cadre de l'autosurveillance des réseaux en France, l'arrêté du 22 juin 2007 impose une estimation des masses de MES rejetées au droit des déversoirs d'orage les plus importants. Il est donc important de mesurer les flux en MES transitant dans le réseau ou déversés dans le milieu naturel. Pour mieux répondre à ces exigences réglementaires, la mesure en continu de la concentration en MES sera essentielle. Les concentrations en 
MES sont classiquement estimées à partir d'analyses en laboratoire effectuées sur des échantillons prélevés in situ durant les événements pluvieux. Cette pratique présente plusieurs inconvénients : transport des échantillons du site au laboratoire, conditionnement et conservation des échantillons, délais pour obtenir les résultats, coût élevé des prélèvements et des analyses, quelques échantillons par événement et quelques événements sont échantillonnés (Bertrand-Krajewski et al., 2008). Il en résulte une faible représentativité temporelle de la dynamique des phénomènes du transport solide dans le réseau et des incertitudes importantes dans l'évaluation des flux annuels des MES (Mourad et al., 2005).

Dans ce contexte, la mesure en continu de la turbidité en réseau d'assainissement commence à se développer car elle permet d'avoir directement accès à la mesure de la dynamique de la pollution particulaire, notamment lors d'événements pluvieux. De récentes études ont montré la possibilité d'utiliser cette mesure pour contrôler, évaluer la qualité des effluents (Langeveld et al., 2005, Lawler et al., 2006) et estimer les flux de MES aux échelles événementielles et annuelle avec une faible dispersion par rapport aux méthodes d'échantillonnage classiques (Lacour et al., 2009a). Il faut noter que l'application de protocoles adaptés permet l'obtention de mesure de la turbidité en continu fiable et précise à des pas de temps courts en réseau d'assainissement (Joannis et al., 2008).

Toutefois, la relation entre la turbidité et la concentration en MES reste une question difficile à traiter (Bertrand Krajewski et al., 2010, Ruban, 1995). En effet, elle dépend de plusieurs paramètres, notamment les caractéristiques géométriques et optiques des particules en suspension qui sont variables et hétérogènes dans les eaux résiduaires ou pluviales urbaines. Ces paramètres sont liés à la nature des effluents, et peuvent varier en temps sec selon les heures et les jours et en temps de pluie en fonction des événements pluvieux. De plus, il faut noter que la turbidité d'une suspension dépend non seulement du type d'effluent mesuré, mais aussi du principe de mesure (atténuation ou néphélométrie) et de la longueur d'onde utilisés. Cette relation n'est donc pas unique ni constante. Cependant, on peut établir des relations moyennes représentatives de différents contextes (par exemple: temps de pluie, temps sec, site spécifique,...) dont la qualité reste assez élevée par exemple à des échelles temporelles comme l'année (Fletcher et Deletic, 2007) et assez efficace pour un site donné.

L'objectif de cet article est de : a) confirmer l'existence d'une relation linéaire entre la turbidité et la concentration en MES, b) étudier et évaluer la variabilité de cette relation sur les deux sites : Cordon Bleu et Saint-Mihiel à Nantes, par temps sec et par temps de pluie et c) évaluer l'effet de cette variabilité sur l'estimation de la concentration en MES, c.à.d. la dispersion de cette concentration en fonction des données d'étalonnage.

Dans un premier temps, nous présentons les bases de données utilisées, puis nous détaillons la méthodologie développée pour évaluer la variabilité de la relation turbidité/MES. Enfin, nous discutons les résultats obtenus.

\section{METHODOLOGIE}

\subsection{Site d'étude}

\subsubsection{Description des sites}

Nantes est l'un des sites de l'observatoire français en hydrologie urbaine (HURRBIS). Les relations entre la turbidité et la concentration en MES ont été suivies par la division Eau et Environnement du Laboratoire Central des Ponts et Chaussées (LCPC) pour deux bassins versants desservis par un réseau d'assainissement unitaire : Saint-Mihiel (SM) et Cordon Bleu (CB) à l'aide de prélèvements échelonnés sur plusieurs années de 1998 à 2006.

Le premier site, SM, est un petit bassin versant d'une surface de 100 hectares situé dans la zone centrale de la ville de Nantes. Le deuxième site, CB, est un bassin versant beaucoup plus étendu (5000 hectares) qui englobe celui de SM.

\subsubsection{Bases de données disponibles}

Par temps sec, nous disposons de six campagnes de mesure de 24 heures à CB et deux campagnes à SM pour établir la relation turbidité-MES. Ces campagnes couvrent l'ensemble des jours de la semaine.

36 événements ont été échantillonnés par temps de pluie à CB et 27 à SM. La turbidité et la concentration en MES ont été mesurées sur ces échantillons. Les périodes de retour de l'intensité de pluie maximale sur 
une heure varient de 3 mois à 10 ans, soit $11 \mathrm{~mm} / \mathrm{h}$ pour une période de retour de 6 mois, $13 \mathrm{~mm} / \mathrm{h}$ pour une période de retour d'un an et $21 \mathrm{~mm} / \mathrm{h}$ pour 10 ans.

La turbidité et la concentration en MES ont été mesurées au laboratoire. La turbidité a été mesurée avec un turbidimètre de marque Ponselle TUI par atténuation de la lumière dans l'infrarouge à $880 \mathrm{~nm}$ en respectant la norme (NF EN ISO 7027, 2000). Ce turbidimètre a été étalonné à la formazine et par conséquent la turbidité est exprimée en Formazin Attenuation Unit (FAU). La concentration en MES a été mesurée en conformité avec la norme (NF EN 872, 2005).

Le temps de pluie a été défini par le critère de la hauteur d'eau dans le collecteur. Les prélèvements de temps de pluie sont réalisés quand la hauteur d'eau dépasse un seuil correspondant à environ 2 fois le débit de pointe de temps sec.

Les couples des valeurs (T, MES) ont été validés et les valeurs atypiques ont été supprimées en utilisant le test statistique «distance de Cook»(Cook, 2000). Le Tableau I résume la base de données MESturbidité :

Tableau I : Inventaire des données MES/Turbidité à CB et SM

\begin{tabular}{|c|c|c|c|c|}
\hline \multirow[b]{3}{*}{ CB } & \multicolumn{2}{|c|}{ Temps de pluie (TP) } & \multicolumn{2}{|c|}{ Temps sec (TS) } \\
\hline & $\mathrm{Nb}$ de pluies & $\mathrm{Nb}$ d'échantillons & $\mathrm{Nb}$ de campagnes & $\mathrm{Nb}$ d'échantillons \\
\hline & 30 & 324 & 6 & 55 \\
\hline SM & 26 & 243 & 2 & 19 \\
\hline
\end{tabular}

\subsection{Relation théorique turbidité/MES}

La turbidité est la réduction de la transparence d'un liquide, dans notre cas les eaux résiduaires ou pluviales urbaines, due à la présence de matières colloïdales et/ou en suspension. La théorie de Mie (Mishendo et al., 1999), pour n classes de particules en suspension homogènes et d'indice de réfraction déterminé permet de démontrer que la relation entre la turbidité mesurée en atténuation $T$ et la concentration en MES $C_{M E S}$ est linéaire:

$$
C_{M E S}=\frac{2}{3}\left(\sum_{i=1}^{n} \frac{Q_{e x t i} p_{m i}}{\rho_{p i} d_{p i}}\right)^{-1} T=a \times T
$$

$Q_{M E S}$ coefficient d'extinction caractéristique de la classe $i$ (il dépend de la longueur d'onde, de l'indice de réfraction, et de la forme des particules); $\rho_{p i}$ masse volumique des particules de la classe $i ; p_{m i}$ fraction des particules de la classe i de diamètre équivalent $d_{p i}$.

Le coefficient de proportionnalité a entre la concentration en particules $C_{M E S}$ et la turbidité $T$ dépend donc des caractéristiques géométriques et optiques des particules en suspension (taille, forme, indice de réfraction, masse volumique,...).

Dans les eaux résiduaires ou pluviales urbaines, les MES ont des caractéristiques à la fois hétérogènes et variables dans le temps, ce qui explique que ce coefficient peut varier en temps sec selon les heures et les jours, et en temps de pluie en fonction des événements pluvieux voire au sein d'un même événement pluvieux.

On notera dans la suite $a_{i j}$ la valeur du rapport entre la concentration en MES et la turbidité associée pour l'échantillon $j$ de l'événement ou la campagne $i$.

\subsection{Méthodes de calcul}

\subsubsection{Analyse de la variabilité du rapport $a_{i j}$}

Nous voulons évaluer la variabilité du rapport $a_{i j}$ en fonction de facteurs tels que les sites, les journées de temps sec, les événements pluvieux. Pour cela on regroupe les observations correspondant à la même occurrence du facteur choisi, et on cherche à évaluer la contribution de ce facteur à la variabilité totale.

Supposons $k$ groupes d'observations constitués (par exemple $k$ événements ou $k$ jours de temps sec ou $k$ sites) $G_{1}, G_{2}, \ldots, G_{k}$ avec respectivement $n_{1}, n_{2}, \ldots, n_{k}$ observations. Les valeurs moyennes peuvent être calculées pour chaque groupe $G_{i}$ et pour l'ensemble des observations :

$$
a_{i}=\frac{1}{n_{i}} \sum_{j} a_{i j} \text { et } a=\frac{1}{\sum_{i} n_{i}} \sum_{i} \sum_{j} a_{i j}
$$

La variabilité totale peut être décomposée en deux termes: une variabilité à l'échelle intergroupe (entre événements par exemple) et une autre à l'échelle intragroupe (lors de l'événement pluvieux par exemple). Cette décomposition s'écrit sous la forme : 


$$
\begin{gathered}
S C_{T}=S C_{\text {int } e r}+S C_{\text {int } r a} \\
\sum_{i=1}^{k} \sum_{j=1}^{n_{i}}\left(a_{i j}-a\right)^{2}=\sum_{i=1}^{k} n_{i}\left(a_{i}-a\right)^{2}+\sum_{i=1}^{k} \sum_{j=1}^{n_{i}}\left(a_{i j}-a_{i}\right)^{2}
\end{gathered}
$$

Le rapport de corrélation donne la part de la variabilité totale des données expliquée par l'effet du facteur groupe :

$$
R^{2}=100 * \frac{S C_{\text {inter }}}{S C_{T}}
$$

C'est un indice qui varie entre 0 et 100. Il tend vers 100 si toute la variabilité observée est expliquée par le facteur qui a présidé à la constitution des groupes.

Cette approche globale a été complétée par une analyse de l'homogénéité des moyennes entre les différents groupes $G_{i}$ au moyen des tests statistiques de significativité (au seuil de $5 \%$ ) en utilisant les tests de Wilcoxon-Mann-Whitney ( $k=2$ : comparaison entre sites) et de Kruskal-Wallis $(k>2$ : comparaison entre les événements pluvieux et les journées de temps sec).

\subsubsection{Evaluation de la concentration en MES : Calculs de régression - Simulation de Monte-Carlo}

Comme les ratios $a_{i j}$ varient, on utilise en pratique des calculs de régression pour convertir les valeurs de turbidité en concentration en MES. Dans un premier temps nous établissons une relation linéaire spécifique à chaque événement pluvieux et à chaque campagne de temps sec en utilisant la régression par moindres carrés ordinaire $C_{M E S}=a T+b$. Ces droites de régression ne passent pas nécessairement par l'origine et admettent donc que les caractéristiques des particules puissent varier au cours d'une journée ou à l'intérieur d'un événement, en supposant que ces variations sont liées à la concentration. Après avoir analysé les variations de ces relations, nous étudierons dans un deuxième temps la possibilité de regrouper tous les événements disponibles pour calculer une régression unique, en mettant l'accent sur l'impact des événements échantillonnés sur l'incertitude de l'évaluation des MES. Pour cela nous faisons l'hypothèse que les événements échantillonnés sont représentatifs des événements susceptibles de se produire sur chaque bassin versant. Donc nous simulons une campagne de mesure virtuelle avec un échantillonnage aléatoire d'un nombre réduit d'événements pluvieux par rapport au nombre d'événements mesurés disponibles pour chaque site. Pour cela, parmi les événements numérotés de 1 à $N$ pour chaque site (Tableau $I$ ), on tire $n \leq N$ événements sans remise et pour chaque jeu d'événements tiré nous calculons une régression turbidité/MES.

Pour chaque valeur de turbidité on obtient donc une distribution de valeurs de la concentration en MES, correspondant à la dispersion des relations moyennes obtenues avec les différents jeux d'événements. Cette distribution nous permet de quantifier la variabilité de la concentration en MES due à la variabilité des données d'étalonnage. Cette variabilité a été exprimée en termes de demi-intervalle de confiance à $95 \%$ car nous avons vérifié, avec le test de normalité de Shapiro-Wilk au seuil de $5 \%$, pour $n \geq 2$, que la distribution des concentrations en MES est normale.

Par ailleurs, pour un jeu d'événements tiré, le modèle de régression implique des erreurs résiduelles. Ces erreurs sont supposées suivre la même loi normale de variance résiduelle $\sigma^{2}$ provenant des résidus observés pour ce jeu. Pour tenir compte de l'influence de ces erreurs, la valeur simulée de la concentration en MES a été calculée en simulant un nombre aléatoire $\tau$ qui suit une loi de Student à $n_{e c h}-2$ degrés de liberté où $n_{e c h}$ est le nombre des échantillons utilisés pour ajuster la droite de régression linéaire. La valeur simulée de MES, pour une turbidité donnée $T_{i}$, sera :

$$
C_{M E S_{\text {simu }}}=C_{M E S_{R L}}+s \sqrt{1+\frac{1}{n_{e c h}}+\frac{\left(T_{i}-m_{T}\right)^{2}}{\sum_{j=1}^{n_{e c h}}\left(T_{j}-m_{T}\right)^{2}}} \tau_{\left(n_{e c h}-2\right)}
$$

Avec $C_{M E S}=a T+b$ la valeur estimée de la concentration en MES par la droite de régression linéaire de paramètres $a$ et $b ; s$ l'estimation de l'écart type résiduel $\sigma ; m_{T}$ la moyenne des turbidités des échantillons $T_{j}$.

La loi de Student a une variance infinie si le nombre de degrés de liberté est plus petit que 3. Pour cette raison, nous avons enlevé les événements dont $n_{e c h}$ inférieur à 5 .

Ce processus est réitéré au maximum 1000 fois pour chaque sous-échantillonnage n donné et pour chaque valeur de turbidité $T_{i}$.

Le choix d'un modèle de régression linéaire ne passant pas par l'origine admet implicitement que ce modèle ne peut pas être extrapolé pour les très faibles valeurs : une turbidité nulle correspondrait à des 
valeurs de la concentration en MES non nulles, voire négatives. De plus, les échantillons de temps de pluie à $\mathrm{CB}$ et SM ont des valeurs de turbidité qui varient seulement entre 100 et $700 \mathrm{FAU}$ qui sont les valeurs les plus probables en réseau d'assainissement unitaire par temps de pluie (Lacour, Joannis et al., 2009b). En conséquence, nous avons effectué le calcul de la concentration en MES pour les valeurs de la turbidité comprises entre 100 et 700 FAU.

\section{RESULTATS ET DISCUSSIONS}

\subsection{Analyse de la variabilité du rapport $a_{i j}$ entre MES et turbidité}

Les rapports $a_{i j}$ sont illustrés sous forme de boîtes à moustaches de Tukey dans la figure $1(a)$; les traits inférieur et supérieur de la boîte correspondent respectivement aux 1er et 3ème quartiles $\left(Q_{1}\right.$ et $\left.Q_{3}\right)$ tandis que la médiane $\left(Q_{2}\right)$ et la moyenne (en rouge) sont représentées par les traits horizontaux à l'intérieur de la boîte. Les deux moustaches inférieure et supérieure délimitent les valeurs dites adjacentes qui sont déterminées à partir de l'écart interquartile $I Q R=Q_{3}-Q_{1}$. Elles valent $Q_{1}-1,5 * I Q R$ et $Q_{3}+1,5 * I Q R$. Les valeurs dites extrêmes situées au-delà des valeurs adjacentes sont individualisées et sont représentées par des marqueurs.

Le site de CB a une dispersion du rapport $a_{i j}$ moins importante que celle du site de SM par temps sec et par temps de pluie. Cette dispersion par temps de pluie donnée par les valeurs adjacentes est de l'ordre de $30 \%$ par rapport à la valeur médiane à $\mathrm{CB}$ et de $40 \%$ à SM. Les valeurs extrêmes de rapport $a_{i j}$ représentent des événements pluvieux décennaux et se caractérisent par des valeurs sensiblement plus élevées que le reste de la population. Pour les autres valeurs on ne décèle pas de tendance en fonction des caractéristiques générales des événements pluvieux concernés. Les valeurs du rapport $a_{i j}$ par temps sec ont une faible dispersion et présentent des valeurs plus faibles que celles de temps de pluie, ce résultat peut s'expliquer par le type des particules transportées par temps de pluie (moins homogènes, plus minérales, ...) (Joannis et al., 2008).

\subsubsection{Variabilité inter-site par temps sec et par temps de pluie}

Les figures $1(a)$ et $(b)$ montrent que le rapport $a_{i j}$ prend des valeurs comparables sur les deux sites par temps sec, ce qui est aussi traduit par le rapport $R^{2}$ égal à $6 \%$ (figure 2). De plus, le test statistique confirme que cette variabilité est non significative du rapport $a_{i j}$ au seuil de $5 \%$. Ce résultat atteste de la relative homogénéité spatiale de ce rapport par temps sec sur les deux sites considérés. Cette homogénéité a également été observée par (Marechal, 2000) sur divers sites par temps sec pour des mesures en atténuation. Il serait intéressant de vérifier ce résultat pour des mesures de la turbidité par néphélométrie. De même par temps de pluie, les figures $1(a)$ et 2 témoignent d'une relative homogénéité de ce rapport sur les deux sites. Cette faible variabilité spatiale peut être également appréciée au moyen de l'analyse statistique. Cette première analyse laisse à penser que la qualité des effluents unitaires par temps de pluie demeure relativement homogène à partir d'une certaine échelle spatiale (Kafi et al., 2008).

\subsubsection{Variabilité par temps sec}

La variabilité entre journées du rapport $a_{i j}$ est non négligeable par rapport à la variabilité au sein de chaque journée. En effet, sur les deux sites, elle représente $40 \%$ à CB et $35 \%$ à SM de la variabilité totale (figure 2). Le test statistique confirme cette variabilité significative du rapport $a_{i j}$ au seuil de $5 \%$.

\subsubsection{Variabilité entre les événements pluvieux}

La variabilité du rapport $a_{i j}$ entre les événements pluvieux est claire. Plus de $70 \%$ de la variabilité totale du rapport $a_{i j}$ par temps de pluie sont dus à la variabilité inter-événementielle (figure 2). Cette variabilité inter-événementielle concorde avec les résultats trouvés par (Chebbo, 1992, Kafi-Benyahia et al., 2005) concernant les caractéristiques des polluants par temps de pluie. Cette variabilité n'a pu être reliée systématiquement aux caractéristiques générales des événements pluvieux telles que la hauteur précipitée, l'intensité, la durée de temps sec antérieure, etc.

\subsection{Qualité de la régression des moindres carrés}

Le tableau II liste l'ensemble des paramètres relatifs aux relations linéaires entre turbidité et MES pour les deux sites SM et $\mathrm{CB}$. Il révèle une forte linéarité de ces relations, la majorité de ces relations ont tendance à passer au voisinage de l'origine, par temps sec et par temps de pluie. De plus, la variabilité de la pente $a$ est similaire de celle du rapport $a_{i j}$ présentée dans la section précédente.

Ces résultats signifient que les variations des caractéristiques des particules au cours d'un événement pluvieux ou d'une journée de temps sec sont insuffisantes pour changer significativement la relation entre 
la turbidité et la concentration en MES. La variabilité limitée de la pente $a$ a une influence sur la relation linéaire construite avec les points de tous les événements pluvieux. En effet, les coefficients de détermination des relations linéaires globales sont un peu plus faibles que la médiane des coefficients de détermination de toutes les relations, mais restent supérieurs à 0.8 .
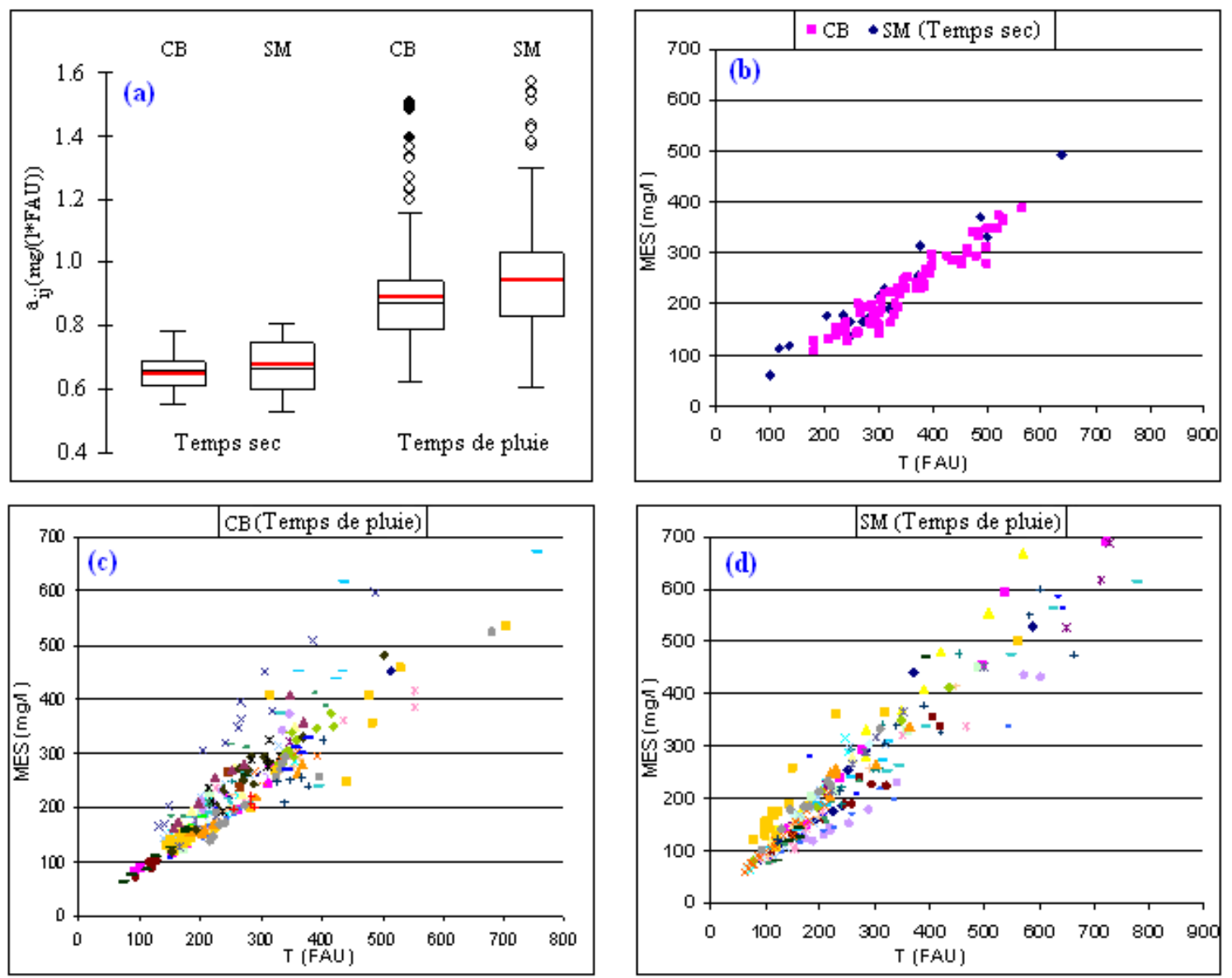

Figure $1 \mid(a)$ : Bô̂tes à moustaches du rapport $a_{i j}$ à SM et CB par temps sec et temps de pluie ; $(b),(c)$, (d) : Relations entre la concentration en MES et la turbidité à CB et à SM par temps sec et par temps de pluie. (Les différents symboles représentent les différents événements)

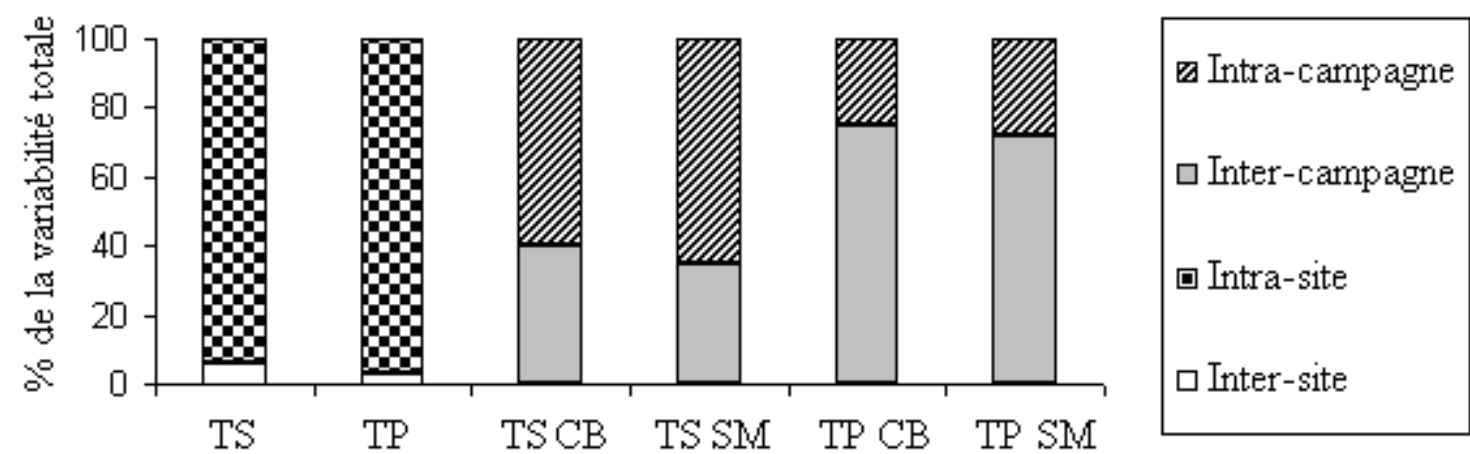

Figure $2 \mid \%$ de la variabilité inter groupe et intragroupe par rapport à la variabilité totale dans le groupe (TS : temps sec, TP : temps de pluie, CB : Cordon Bleu, SM : Saint-Mihiel)

Incertitudes de la concentration en MES évaluée à partir de la turbidité

La figure 3 montre les demi-intervalles de confiance à $95 \%$ en pourcentage de la concentration en MES, pour les deux sites CB et SM. L'impact du nombre d'événements utilisés pour l'étalonnage de la relation turbidité - MES sur la dispersion de la concentration en MES est net. La concentration en MES à CB se distingue par une dispersion plus importante en raison de la présence de deux événements décennaux qui 
se distinguent nettement des autres événements en terme de relations d'étalonnage (figure 1 (c)). Par exemple, pour évaluer la concentration en MES à partir de la mesure de la turbidité avec une dispersion de l'ordre de $\pm 20 \%$, dans l'intervalle [150, 700] FAU, il faut échantillonner au moins 5 événements dans l'année, un nombre d'événements qui semble compatible avec les durées des campagnes de mesures réalisées dans le cadre des études de diagnostic des réseaux d'assainissement. Ce qui montre que l'utilisation de la turbidité apporte un gain de précision important dans l'évaluation de la concentration en MES par temps de pluie.

Tableau II | Paramètres relatifs aux relations entre turbidité et $M E S\left(C_{M E S}=a T+b\right)$ pour les bases de données de CB et de SM par temps de pluie et par temps sec

\begin{tabular}{ccccc}
\hline & & & CB & SM \\
\hline & $\begin{array}{c}\text { Coefficient de } \\
\text { détermination }\end{array} r^{2}$ & Médiane & 0.92 & 0.97 \\
\cline { 2 - 4 } & $\begin{array}{c}\text { Ordonnée à l'origine } \\
b(m g / l)\end{array}$ & IC empirique à 95\% & {$[0.74 ; 0.98]$} & {$[0.80 ; 0.99]$} \\
\cline { 2 - 4 } Temps de \\
\cline { 2 - 4 } & $\begin{array}{c}\text { Pente } a \\
(m g . /(l . F A U))\end{array}$ & Médiane & 0 & -5 \\
\cline { 2 - 4 } & $r^{2}$ & IC empirique à 95\% & {$[-29 ; 35]$} & {$[-42 ; 30]$} \\
\cline { 2 - 4 } & $b \pm s_{b}$ & Médiane & 0.82 & 0.90 \\
\cline { 2 - 4 } & $a \pm s_{a}$ & Relation globale & 0.82 & 0.82 \\
\cline { 2 - 4 } & $\mathrm{r}^{2}$ & Relation globale & $15 \pm 7$ & $7 \pm 5$ \\
\cline { 2 - 5 } Temps sec & $b \pm s_{b}$ & Relation globale & $0.85 \pm 0.02$ & $0.89 \pm 0.02$ \\
\cline { 2 - 5 } & $a \pm s_{a}$ & Relation globale & $-8 \pm 9$ & 0.93 \\
\cline { 2 - 5 } & & Relation globale & $0.71 \pm 0.02$ & $0.72 \pm 0.05$ \\
\hline
\end{tabular}
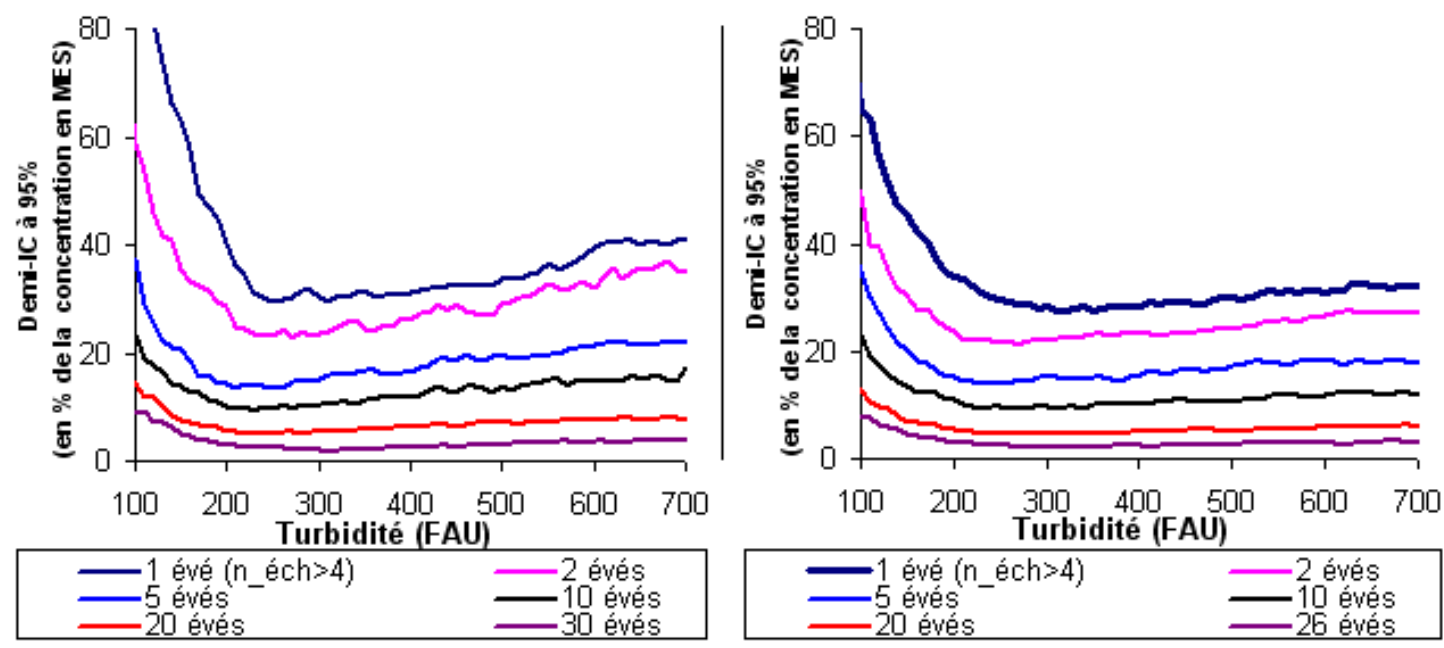

Figure 3 | Dispersion de la concentration en MES par temps de pluie en fonction de la turbidité et du nombre d'événements échantillonnés pris en compte pour les deux sites CB et SM

\section{CONCLUSION}

Cette étude confirme l'intérêt de l'utilisation de la turbidité pour analyser la dynamique particulaire dans un réseau d'assainissement unitaire. Les principaux points à retenir sont les suivants :

- Le rapport entre la concentration en MES et la turbidité varie de 0.5 à $0.8(m g . /(l . F A U))$ par temps sec et de 0.6 à $1.4(\mathrm{mg} . /(\mathrm{l} . \mathrm{FAU}))$ pour la plupart des événements pluvieux ;

- La variabilité de ce rapport entre les deux sites étudiés n'est pas significative au seuil de $5 \%$, que ce soit par temps sec ou par temps de pluie ;

- La variabilité de $a_{i j}$ est non négligeable d'une journée de temps sec à une autre mais reste inférieure à la variabilité interne aux journées ;

- La variabilité inter-événementielle du rapport $a_{i j}$ est supérieure à la variabilité interne aux événements pluvieux ; 
- Les relations d'étalonnage MES-turbidité sont linéaires et la majorité des relations de temps de pluie a tendance à passer au voisinage de l'origine ;

- Pour un objectif opérationnel : 5 événements suffisent à établir une relation MES-turbidité permettant d'évaluer des valeurs instantanées de concentration en MES de temps de pluie dans l'intervalle de turbidité [150, 700] FAU avec une incertitude inférieure à $20 \%$.

Ces bons résultats peuvent être expliqués par la variabilité inter-événementielle assez modérée des relations linéaires d'étalonnage MES-turbidité. Cette faible variabilité permet d'utiliser la mesure en continu de turbidité pour appréhender les phénomènes de transport solide dans le réseau, notamment les processus de sédimentation / érosion. Il serait intéressant de poursuivre ces recherches sur d'autres sites pour les généraliser.

\section{REMERCIEMENTS}

Cette étude a été réalisée dans le cadre du programme de recherche OPUR. Les auteurs remercient pour leur contribution: SEPIA Conseils, la municipalité de Paris, l'Agence de l'Eau Seine Normandie (AESN), le Syndicat Interdépartemental de l'Assainissement de l'Agglomération Parisienne (SIAAP), la direction de l'eau et de l'assainissement du département de la Seine Saint Denis (DEA93), la direction des services de l'eau et de l'assainissement du Val de Marne (DSEA94), le Conseil Régional d'Île-de-France (CRIF) et la communauté urbaine Nantes Métropole.

\section{BIBLIOGRAPHIE}

ASHLEY, R., BERTRAND-KRAJEWSKI, J.L. AND HVITVED-JACOBSEN, T. (2005) Sewer solids 20 years of investigation. Water Science and Technology 52 (3), 73-84.

BERTRAND-KRAJEWSKI, J.L., BARRAUD, S., KOUYI, G.L., TORRES, A. AND LEPOT, M. (2008) On-line monitoring of particulate pollutant loads in urban sewer systems : stakes, methods, example of application. La Houille Blanche (4), 49-57.

BERTRAND KRAJEWSKI, J.L., JOANNIS, C., CHEBBO, G., RUBAN, G., METADIER, M. AND LACOUR, C. (2010) Comment utiliser la turbidité pour estimer en continu les concentrations en MES et/ou DCO: Une approche méthodologique pour les réseaux d'assainissement. TSM (1-2), pp. 36-46.

CHEBBO, G. (1992) Solides des rejets pluviaux urbains. Caractérisation et traitabilité. Thèse de doctorat, Ecole Nationale des Ponts et Chaussées, Paris, France.

CHEBBO, G., BACHOC, A., LAPLACE, D. AND LEGUENNEC, B. (1995) The transfer of solids in combined sewer networks. Water Science and Technology 31 (7), 95-105.

COOK, R.D. (2000) Detection of influential observation in linear regression. Technometrics 42(1), 65-68.

ELLIS, J.B. AND HVITVED JACOBSEN, T. (1996) Urban drainage impacts on receiving waters. Journal of Hydraulic Research 34 (6), 771-783.

FLETCHER, T.D. AND DELETIC, A. (2007) Statistical evaluation and optimisation of stormwater quality monitoring programmes. Water Science and Technology 56 (12), 1-9.

GROMAIRE, M.C., GARNAUD, S., SAAD, M. AND CHEBBO, G. (2001) Contribution of different sources to the pollution of wet weather flows in combined sewers. Water Research 35 (2), 521533.

JOANNIS, C., RUBAN, G., GROMAIRE, M.C., BERTRAND-KRAJEWSKI, J.L. AND CHEBBO, G. (2008) Reproducibility and uncertainty of wastewater turbidity measurements. Water Science and Technology 57(10), 1667-1673.

KAFI-BENYAHIA, M., GROMAIRE, M.G. AND CHEBBO, G. (2005) Spatial variability of characteristics and origins of urban wet weather pollution in combined sewers. Water Science and Technology 52 (3), 53-62.

KAFI, M., GASPERI, J., MOILlERON, R., GROMAIRE, M.C. AND CHEBBO, G. (2008) Spatial variability of the characteristics of combined wet weather pollutant loads in Paris. Water Research 42(3), 539-549.

LACOUR, C., JOANNIS, C. AND CHEBBO, G. (2009a) Assessment of annual pollutant loads in combined sewers from continuous turbidity measurements: Sensitivity to calibration data. Water Research 43 (8), 2179-2190.

LACOUR, C., JOANNIS, C., GROMAIRE, M.C. AND CHEBBO, G. (2009b) Potential of turbidity monitoring for real time control of pollutant discharge in sewers during rainfall events. Water Science and Technology 59 (8), 1471-1478. 
LANGEVELD, J.G., VELDKAMP, R.G. AND CLEMENS, F. (2005) Suspended solids transport: an analysis based on turbidity measurements and event based fully calibrated hydrodynamic models. Water Science and Technology 52 (3), 93-101.

LAWLER, D.M., PETTS, G.E., FOSTER, I.D.L. AND HARPER, S. (2006) Turbidity dynamics during spring storm events in an urban headwater river system: The Upper Tame, West Midlands, UK. Science of the Total Environment 360 (1-3), 109-126.

MARECHAL, A. (2000) Relations entre caractéristiques de la pollution particulaire et paramètres optiques dans les eaux résiduaires urbaines. Thèse de doctorat, Institut national polytechnique de Lorraine, Vandoeuvre-lès-Nancy, France.

MISHENDO, M.I., HOVENIER, J.W. AND TRAVIS, L.D. (1999) Light Scattering by Nonspherical Particules: Theory, Measurements, and Applications. San Diego, Academic Press, 620 p.

MOURAD, M., BERTRAND-KRAJEWSKI, J.L. AND CHEBBO, G. (2005) Sensitivity to experimental data of pollutant site mean concentration in stormwater runoff. Water Science and Technology 51 (2), 155-162.

NF EN 872 (2005). Qualité de l'eau - Dosage des matières en suspension - Méthode par filtration sur filtre en fibres de verre. Paris (France): AFNOR, Juin 2005, 14 p.

NF EN ISO 7027 (2000) Qualité de l'eau-détermination de la turbidité, AFNOR, Paris,France, 14 p.

RUBAN, G. (1995) Continuous measurement of pollution due to urban effluents under wet conditions using optical-systems. Water Science and Technology 32(1), 241-247.

SAGET, A., CHEBBO, G. AND DESBORDES, M. (1995) Urban discharges during wet weather - what volumes have to be treated. Water Science and Technology 32(1), 225-232.

SMULLEN, J.T., SHALLCROSS, A.L. AND CAVE, K.A. (1999) Updating the US nationwide urban runoff quality data base. Water Science and Technology 39(12), 9-16. 